\title{
Dynamic Feature Extraction for Speech Signal Based On MUSIC and Modulation Spectrum
}

\author{
Han Zhiyan and Wang Jian \\ College of Engineering, Bohai University, No. 19, Sci-tech Road, High \& New Technological and Industrial \\ Development District, Jinzhou, China, 121000
}

(Received 19 August 2015; accepted 30 June 2016)

\begin{abstract}
A novel dynamic feature extraction algorithm is proposed to help improve speech recognition robustness in noise environmental conditions. Owing to the modulation spectrum having time-frequency agglomeration performance, according to different reflections in the modulation spectrum for interference and speech signals, we first calculate the Multiple Signal Classification (MUSIC) spectrum and then get the modulation spectrum. We then filter the modulation spectrum signal. For the filter signal, use a 32 frames signal is used as a processing unit to get the modulation spectrum energy vector. This reveals the close correlation between the speech signal frames and can well reflect speech dynamic characteristics. Finally, the cepstrum coefficients are extracted as the feature parameter. It not only adequately reflects speech dynamic characteristics, but also has lower sensitivity for the speech environment. The effectiveness of the feature is discussed in view of the class separability and speaker variability properties. We evaluated the feature under different kinds of noise (white noise, pink noise, street noise, and panzer noise) and different signal-to-noise ratios $(-5 \mathrm{~dB}, 0 \mathrm{~dB}, 5 \mathrm{~dB}, 10 \mathrm{~dB}$, and $15 \mathrm{~dB})$. The experimental results show that the novel feature has good robustness and computational efficiency under low signal-to-noise ratios and plays a very good foreshadowing role in latter speech research.
\end{abstract}

\section{NOMENCLATURE}

$\begin{array}{ll}\text { MFCC: } & \text { Mel Frequency Cepstral Coefficient } \\ \text { MUSIC: } & \begin{array}{l}\text { Modulation Spectrum; Multiple Signal } \\ \text { Classification }\end{array} \\ \text { LPC: } & \text { Linear Prediction Coefficients } \\ \text { LSP: } & \text { Linear Spectrum Pair } \\ \text { FFT: } & \text { Fast Fourier Transform } \\ \text { LDA: } & \text { Linear Discriminant Analysis } \\ \text { DM: } & \text { Determinant Measure } \\ \text { HMM: } & \text { Hidden Markov Model } \\ \text { BPNN: } & \text { Back-Propagation Neural Networks } \\ \text { SNR: } & \text { Signal-to-Noise Ratio }\end{array}$

\section{INTRODUCTION}

Research on the robustness of speech recognition was still a challenging task, especially in the development of core speech processing algorithms. One example was feature extraction from speech signals. Namely, extracting features that could reflect speech characteristics from the speech waveforms. It not only could reduce the number of calculations and storage, but could also filter out useless and redundant information and was one of the most fundamental and important aspects of speech recognition. Some time domain features, such as amplitude feature, short-time frame average energy, shorttime frame zero crossing rate, short-time autocorrelation coefficient, et cetera appeared. With the development of recognition technology, we found that the stability and separating capacity for the time domain features were not good, so we began using the frequency domain feature as a speech feature, such as pitch period, formant frequency, linear prediction coefficients (LPC), linear spectrum pair (LSP), cepstrum coefficient, and so on. Among them, the MFCC, which was based on the auditory model, was a widely-applied feature at present. However, once these features were used in the noise environment, their performance droped sharply. ${ }^{1-4}$

The features mentioned above reflect the static feature of speech signal, while a dynamic feature was part of speech diversity, which was different from a stationary random process. It had time correlation, and revealed the close relationship between speech signal (pre and post). We could get the dynamic feature by differential parameters and acceleration parameters for the static feature. However, differential parameters and acceleration parameters could not fully dig out dynamic information. Therefore, studying the dynamic feature of the speech signal was an inevitable trend to improve the performance of speech recognition.

Estimating the time-varying spectrum was a key first step in the speech feature extraction. ${ }^{5-10}$ MFCC was computed by applying a Mel-scaled filter bank either to the short-time Fast Fourier Transform (FFT) magnitude spectrum or to the short-term LPC-based spectrum. However, both FFT and LPCbased spectrum were very sensitive to noise contamination. Eigenvector-based methods, such as MUSIC, were popular in sinusoidal frequency estimation due to their high resolution and less prior information. Moreover, MUSIC algorithm had well noise restraining ability. We adopted the MUSIC spectrum instead of the traditional method. 
Owing to the modulation spectrum having time-frequency agglomeration performance, it not only adequately reflected speech dynamic characteristics, but also had lower sensitivity for the speech environment. In the modulation spectrum, the interference component produced by additive noise and channel distortion eased to separate from speech signal. Which is why we used modulation spectrum technology in this study to extract feature, which will be better.

\section{ALGORITHM DESCRIPTION}

\subsection{MUSIC Algorithm}

According to matrix theory, linear space $\mathbf{v}_{1}, \mathbf{v}_{2} \ldots \mathbf{v}_{N}$, could decompose into a direct sum of two subspaces $\mathbf{V}_{1}$ and $\mathbf{V}_{2}$, that is: ${ }^{11-13}$

$$
\begin{aligned}
\mathbf{V}_{1} & =\left[\mathbf{v}_{1}, \mathbf{v}_{2}, \ldots \mathbf{v}_{p}\right] \\
\mathbf{V}_{2} & =\left[\mathbf{v}_{p+1}, \mathbf{v}_{p+2}, \ldots \mathbf{v}_{N}\right] ;
\end{aligned}
$$

then:

$$
\mathbf{V}=\left[\begin{array}{ll}
\mathbf{V}_{1} & \mathbf{V}_{2}
\end{array}\right]
$$

For the signal consisting of $p$ sinusoidal components, the complex amplitudes and frequencies were $g_{k}, \omega_{k}, k=$ $1,2, \ldots p$ respectively, then the signal could be expressed as:

$$
\mathbf{X}=\mathbf{S g}+\mathbf{W}
$$

where $\mathbf{W}$ was white noise with a zero mean and a variance $\sigma^{2}, \mathbf{X}^{T}=\left[\mathbf{X}_{1}, \mathbf{X}_{2}, \ldots \mathbf{X}_{N}\right], \mathbf{S}=\left[e\left(\omega_{1}\right), e\left(\omega_{2}\right), \ldots e\left(\omega_{p}\right)\right]$, $\mathbf{e}^{T}\left(\omega_{k}\right)=\left[1, e^{j \omega_{k}}, \ldots e^{j(N-1) \omega_{k}}\right], \mathbf{g}^{T}=\left[g_{1}, g_{2}, \ldots g_{p}\right]$, $\mathbf{W}^{T}=\left[w_{1}, w_{2}, \ldots w_{N}\right]$.

The autocorrelation matrix for signal $\mathbf{X}_{n}, n=1,2, \ldots N$ can be shown as:

$$
\mathbf{R}_{X X}=E\left[\mathbf{X X}^{H}\right]=\mathbf{S G S}^{H}+\sigma^{2} \mathbf{I}
$$

where $\mathbf{G}$ was the autocorrelation matrix with $p$ sinusoidal components.

According to the matrix theory, eigenvalue decomposition for Eq. (5) was:

$$
\mathbf{R}_{X X}=\mathbf{V} \boldsymbol{\Lambda} \mathbf{V}^{H}=\sum_{i=1}^{N} \lambda_{i} \mathbf{v}_{i} \mathbf{v}_{i}^{H}
$$

where $\mathbf{V}=\left[\mathbf{v}_{1}, \mathbf{v}_{2}, \ldots \mathbf{v}_{N}\right], \boldsymbol{\Lambda}=\operatorname{diag}\left[\lambda_{1}, \lambda_{2}, \ldots \lambda_{N}\right]$.

MUSIC algorithm was a noise subspace frequency estimator; the weighted spectrum estimation is as follows:

$$
P(\omega)=\sum_{i=p+1}^{N} \mathbf{u}_{k}\left|\mathbf{e}^{H}(\omega) \mathbf{v}_{i}\right|^{2} ;
$$

where $\mathbf{v}_{i}, i=p+1, p+2, \ldots N$ was the eigenvector corresponding to noise subspace and $u_{k}$ was a group of positive weights, $\mathbf{e}^{H}=\left[1, e^{j \omega}, \ldots e^{j(N-1) \omega}\right]$. Notice here, when $\omega=\omega_{i}, P\left(\omega_{i}\right)=0, i=1,2, \ldots p$, the reciprocal of $P(\omega)$ was the peak function in the frequency domain. This provided a method for sinusoidal component frequency estimation. So the power spectrum function was as follows:

$$
P_{\text {MUSIC }}(\omega)=\frac{1}{\sum_{i=p+1}^{N}\left|\mathbf{e}^{H}(\omega) \mathbf{v}_{i}\right|^{2}} .
$$

According to the process of the MUSIC algorithm, we could see that the mathematical principle of this algorithm was through singular value decomposition for the signal autocorrelation matrix. The signal eigenspace was decomposed into signal subspace relative to the signal component and noise subspace orthogonal to the signal component, and then estimated signal frequencies using the orthogonality of the two spaces. So, in theory, this method had the advantages that FFT could not substitute. Therefore, it was called the super-resolution frequency estimation algorithm.

\subsection{Modulation Spectrum Principle}

The definition of speech modulation spectrum is as follows:

$$
\begin{array}{r}
M(\omega, \eta)= \\
\frac{1}{2 \pi} \int_{-\infty}^{\infty} \int_{-\infty}^{\infty} x\left(t+\frac{\tau}{2}\right) x^{*}\left(t-\frac{\tau}{2}\right) e^{-j \omega \tau} e^{-j \eta \tau} d \tau d t= \\
X^{*}\left(\omega-\frac{\eta}{2}\right) X\left(\omega+\frac{\eta}{2}\right) ;
\end{array}
$$

where $\tau$ is delay time, $\omega$ and $\eta$ are speech frequency and modulation spectrum respectively.

$M(\omega, \eta)$ could be considered as the two dimensional transform of the autocorrelation function $x(t)$ or as the autocorrelation function for $X(\omega)$. However, there was also some unwanted coherent term. So we had to smooth process on the modulation spectrum $M(\omega, \eta)$ via short window. We usually choose a Hamming window as the window function. After smoothing, the modulation spectrum is as follows:

$$
M^{s p}(\omega, \eta)=M_{W}(\omega, \eta) * M_{x}(\omega, \eta)
$$

where $M_{W}(\omega, \eta)$ is the modulation spectrum for window function $W(t)$.

Figure 1 and Fig. 2 show the improvements of clean speech recognition rate and noise speech recognition rate on different modulation spectrum frequency bands respectively. ${ }^{14-16}$

The figures, from the first section to the thirteenth section express the frequency in the range of $0-0.5 \mathrm{~Hz}, 0.5-1 \mathrm{~Hz}, 1-$ $1.4 \mathrm{~Hz}, 1.4-2 \mathrm{~Hz}, 2-2.8 \mathrm{~Hz}, 2.8-4 \mathrm{~Hz}, 4-5.7 \mathrm{~Hz}, 5.7-8 \mathrm{~Hz}, 8-$ $11.3 \mathrm{~Hz}, 11.3-16 \mathrm{~Hz}, 16-22.6 \mathrm{~Hz}, 22.6-32 \mathrm{~Hz}$, and $32-40 \mathrm{~Hz}$ respectively.

From Figs. 1 and 2, we can see that almost all the useful features for speech recognition were concentrated on 1-13 Hz. Among them, the characteristics around $4 \mathrm{~Hz}$ were the most important. We could also see that all of the modulation components were useful in a clean speech environment, but in a noise speech environment, the modulation components under 


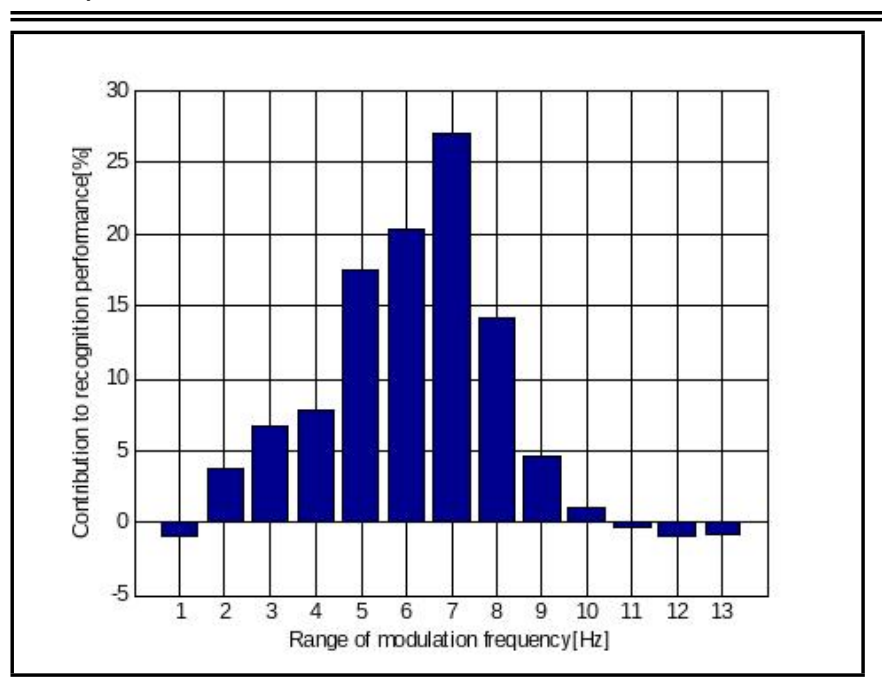

Figure 1. The improvement of recognition accuracy by including the modulation frequency for clean speech.

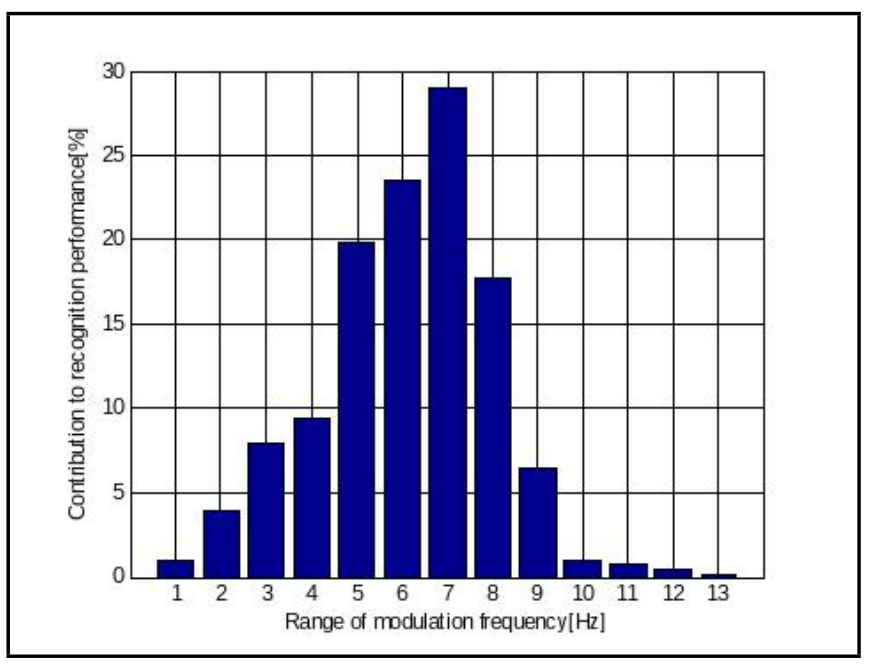

Figure 2. The improvement of recognition accuracy by including the modulation frequency for noise speech.

$0.5 \mathrm{~Hz}$ and over $16 \mathrm{~Hz}$ did not affect the recognition rate; if we had used them, the recognition rate would have decreased.

According to the characteristics of the modulation spectrum, we designed a band-pass filter to suppress interfering components with passband of $1-13 \mathrm{~Hz}$. The transfer function was as follows:

$$
H(z)=0.1 z^{4} \frac{2+z^{-1}+z^{-3}-2 z^{-4}}{1-0.98 z^{-1}} .
$$

The frequency response is shown in Fig. 3.

\section{DF-MMS FEATURE EXTRACTION}

The new feature was called DF-MMS. Figure 4 shows a schematic diagram of the DF-MMS.

We assumed that $x(n)$ denoted an input speech signal the proposed DF-MMS algorithm could be summarized as follows:

Step 1: Speech signal $x(n)$ underwent a series of preprocessing, which included preemphasis, frame, window interception and endpoint detection.

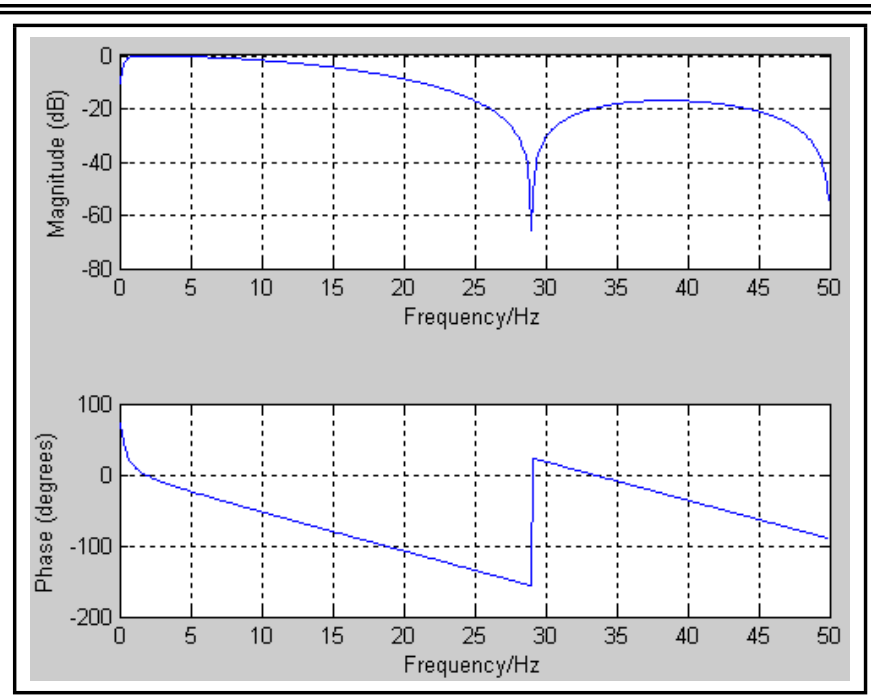

Figure 3. The frequency response for filter.

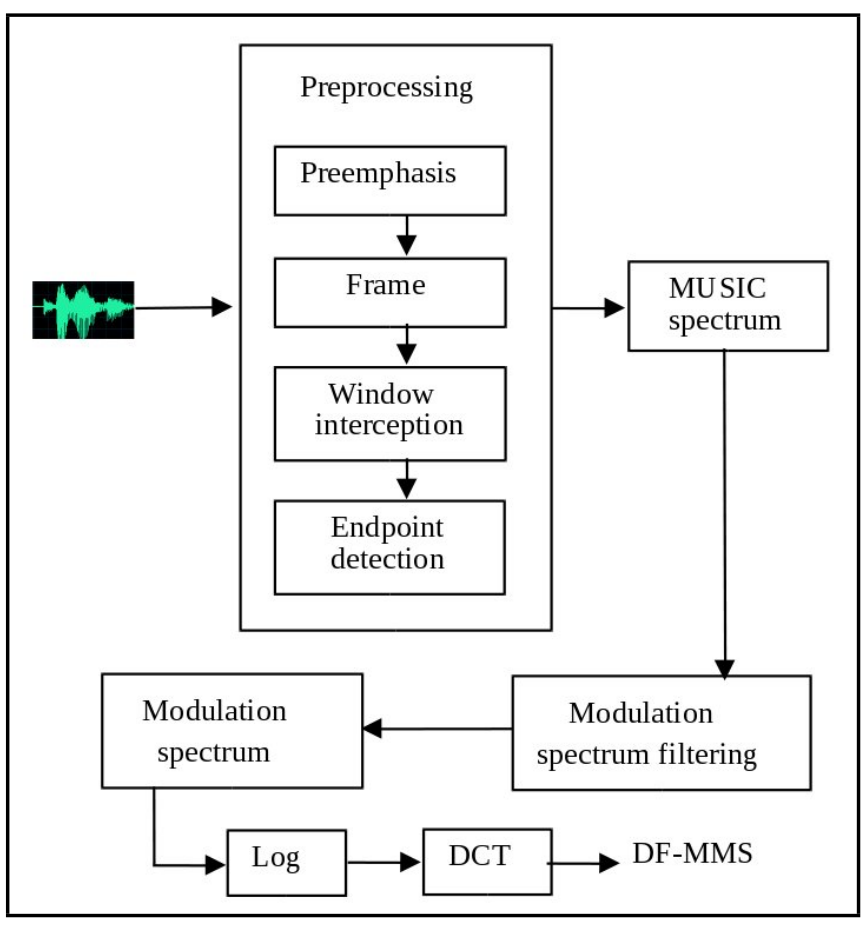

Figure 4. A schematic diagram of the DF-MMS.

Step 2: Calculated the MUSIC spectrum using Eq. (8).

$$
P_{\text {MUSIC }}(\omega)=\frac{1}{\sum_{i=p+1}^{N}\left|\mathbf{e}^{H}(\omega) \mathbf{v}_{i}\right|^{2}} .
$$

Step 3: Let speech MUSIC spectrum at the time $t$ be $P_{\text {MUSIC }}(t, \omega)$, then the modulation spectrum will be:

$$
M^{s p}(\omega, \eta)=\int P_{\text {MUSIC }}(t, \omega) W(t) e^{-j \eta t} d t .
$$

The filter of Eq. (11) was then used to suppress interfering components for the modulation spectrum and get the signal spectrum $F(\omega, \eta)$.

Step 4: For the molulation spectrum filtering signal $F(\omega, \eta)$, 32 frames signal was used as a processing unit to get a $F \times$ 32 matrix. The sum of squares of the same row data were 
then calculated. We could get a vector, that is, the modulation spectrum energy vector $t(i), 0 \leq i \leq F, F$ was frame size.

Step 5: Calculated the Log for the front $F / 2+1$ features.

$$
\mathbf{u}(i)=\log (\mathbf{t}(i)), \quad i=0,1, \ldots F / 2 .
$$

Step 6: Took the DCT.

$$
\mathbf{c}[n]=\cos \left[n \cdot(i+0.5) \cdot \frac{\pi}{F / 2+1}\right] * \mathbf{u}(i)
$$

where $i=0,1, \ldots F / 2,1 \leq n \leq L ; L$ was the desired order of DF-MMS.

\section{EXPERIMENT AND RESULT ANALYSIS}

\subsection{Class Separability and Interspeaker Variability}

The effectiveness of a feature extraction scheme depended mainly on how well separated the different speech classes and suppress speaker dependent information were. We described a measure based on the linear discriminant analysis (LDA) technique in order to analyze class separability and interspeaker variability .

\subsubsection{Class separability analysis}

Class separability could be measured by using Fishers LDA. ${ }^{17}$ The class mean $\mathbf{m}_{c}$ and global mean $\mathbf{m}$ were computed as:

$$
\begin{aligned}
\mathbf{m}_{c} & =\frac{1}{N_{c}} \sum_{i=1}^{N_{c}} \mathbf{x}_{c, i} \\
\mathbf{m} & =\frac{1}{N} \sum_{c=1}^{C} \sum_{i=1}^{N_{c}} \mathbf{x}_{c, i}
\end{aligned}
$$

where $\mathbf{x}_{c, i}, c=1,2, \ldots C, i=1,2, \ldots N_{c}$ was $D$ dimensional feature vectors for speech class $c . N=\sum_{c=1}^{C}$ stood for the total sample numbers.

The within-class scatter matrices $\mathbf{S}_{W}$ and the between-class scatter matrices $\mathbf{S}_{B}$ were computed as:

$$
\begin{aligned}
\mathbf{S}_{W} & =\frac{1}{N} \sum_{c=1}^{C} \sum_{i=1}^{N_{c}}\left(\mathbf{x}_{c, i}-\mathbf{m}_{c}\right)\left(\mathbf{x}_{c, i}-\mathbf{m}_{c}\right)^{T} \mathbf{x}_{c, i} ; \\
\mathbf{S}_{B} & =\frac{1}{N} \sum_{c=1}^{C} N_{c}\left(\mathbf{m}_{c}-\mathbf{m}\right)\left(\mathbf{m}_{c}-\mathbf{m}\right)^{T} .
\end{aligned}
$$

We considered a linear projection of the feature vectors onto a $d$ dimensional subspace, where $d \ll D$. The projection matrix $\mathbf{A}$ was chosen so that the projected feature vectors belonged to one class. This was achieved by computing a projection that maximized the objective function of the ratio between the within-class variance and the between-class variance in the projected space:

$$
\mathbf{A}=\underset{L}{\operatorname{argmax}} \frac{\left|\mathbf{L} \mathbf{S}_{B} \mathbf{L}^{T}\right|}{\left|\mathbf{L} \mathbf{S}_{W} \mathbf{L}^{T}\right|}
$$

The columns of $\mathbf{A}$ were the eigenvectors corresponding to the largest eigenvalues of $\mathbf{S}_{W}^{-1} \mathbf{S}_{B}$. The optimum value of objective function was the product of the $d$ largest eigenvalues. We used the logarithm of product as the Determinant Measure (DM):

$$
D M=\log \left(\prod_{i=1}^{d} \lambda_{i}\right)
$$

We used the Hidden Markov Model (HMM) stated as the speech classes. For all the features, we generated $D=$ 96 dimensional feature vectors by splicing eight consecutive frames. These feature vectors were then projected onto a $d=30$-dimensional subspace using LDA. The DM values for DF-MMS and differential parameter for MFCC were -70.42 and -83.06 respectively. The results indicated that DF-MMS achieved the best class separability among all schemes considered.

\subsubsection{Interspeaker variability analysis}

We used a simple modification of the LDA method to evaluate the robustness of speaker variability. ${ }^{17}$ The following equation was used to define the mean of class $c$ for speaker $s$ :

$$
\mathbf{m}_{c, s}=\frac{1}{N_{c, s}} \sum_{i=1}^{N_{c, s}} \mathbf{x}_{c, s, i}
$$

where $\mathbf{x}_{c, s, i}, c=1,2, \ldots C, s=1,2, \ldots S, i=1,2, \ldots N_{c, s}$ was $D$ dimensional feature vector for speech class $c$ and speaker $s$. Let $M_{c}$ denote the total number of speakers that had samples in class $c, N_{c, s} \neq 0$. For the large databases, all the speakers had samples from all classes. Hence, $M_{c}$ will equal to $S$. We can now compute the class means as:

$$
\mathbf{m}_{c}=\frac{1}{M_{c}} \sum_{s} \mathbf{m}_{c, s} .
$$

The global mean for the entire database was computed as:

$$
\mathbf{m}=\frac{1}{M} \sum_{c=1}^{C} M_{c} \mathbf{m}_{c} ;
$$

where $M$ was the total number of such means.

The between-class $\mathbf{S}_{B}$ and the within-class $\mathbf{S}_{W}$ scatter matrices were computed as:

$$
\begin{aligned}
\mathbf{S}_{W} & =\frac{1}{M} \sum_{c=1}^{C} \sum_{s}\left(\mathbf{m}_{c, s}-\mathbf{m}\right)\left(\mathbf{m}_{c, s}-\mathbf{m}\right)^{T} \\
\mathbf{S}_{B} & =\frac{1}{M} \sum_{c=1}^{C} M_{c}\left(\mathbf{m}_{c}-\mathbf{m}\right)\left(\mathbf{m}_{c}-\mathbf{m}\right)^{T}
\end{aligned}
$$

Using the above expressions for $\mathbf{S}_{B}$ and $\mathbf{S}_{W}$, the DM values for DF-MMS and differential parameter for MFCC were -63.85 and -80.94 respectively. The results indicated that DF-MMS had the largest DM. 


\subsection{Recognition Efficiency}

Some experiments were conducted to evaluate the proposed feature for speech recognition system performance. They could be considered in two aspects: recognition rate and computational consideration. The recognition rate was evaluated under different noises (white noise, pink noise, street noise, and panzer noise) and different SNR $(-5 \mathrm{~dB}, 0 \mathrm{~dB}, 5 \mathrm{~dB}, 10 \mathrm{~dB}$, and $15 \mathrm{~dB}$ ). The data was recorded at $11.025 \mathrm{kHz}$ sampling rate and coded into 256 sampling points with a frame shift of 80 sampling points, where each frame was represented by a 13 dimension vector. We used improved Back-Propagation Neural Networks (BPNN) as the classifier and binary coding output to improve the real-time performance. ${ }^{18}$

The noisy speech data was derived by adding noisy data into the clean speech signal. Because the noise could be divided into additive noise and multiplicative noise, the multiplicative noise could be transformed to additive noise through homomorphic transform. Therefore, discussing additive noise had representative. In this paper, we used global SNR as follows:

$$
S N R=10 \log \frac{\sum_{k=0}^{N-1} s^{2}(k)}{\sum_{k=0}^{N-1} n^{2}(k)} ;
$$

where $s(k)$ was the clean speech signal through the endpoint detector, and was $n(k)$ was the noisy speech signal.

Figures 5(a)-(d) show the recognition performance curves of using DF-MMS and differential parameter for MFCC under white noise, pink noise, street noise, and panzer noise respectively.

From Figs. 5(a)-(d), we can see that the feature DF-MMS improved the recognition rate and robust performance compared with a differential parameter for MFCC under low SNR. This was because we used MUSIC spectrum and the modulation spectrum. In particular, the use of the modulation spectrum not only reflected speech dynamic characteristics, but also had lower sensitivity for the speech environment. And we used a 32 frames signal as a processing unit to get the modulation spectrum energy vector, which revealed the close correlation between the speech signal frames could well reflect speech dynamic characteristics.

\section{CONCLUSIONS}

In this paper, we proposed a robust speech feature extraction method based on MUSIC and the modulation spectrum. The results show that significant advances have been achieved in this area. Although feature DF-MMS can be seen to be modest increase over the differential parameter for MFCC, it compensates for the loss in the search and its kernel algorithm has been used in the voice controlled system of a company.

\section{ACKNOWLEDGMENTS}

This work was supported by a grant from the National Natural Science Foundation of China (No. 61403042, No.

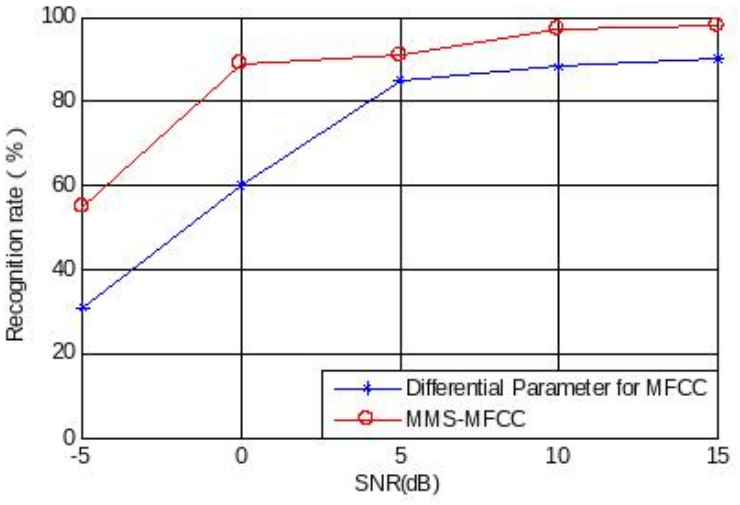

(a) The recognition curve under white noise.

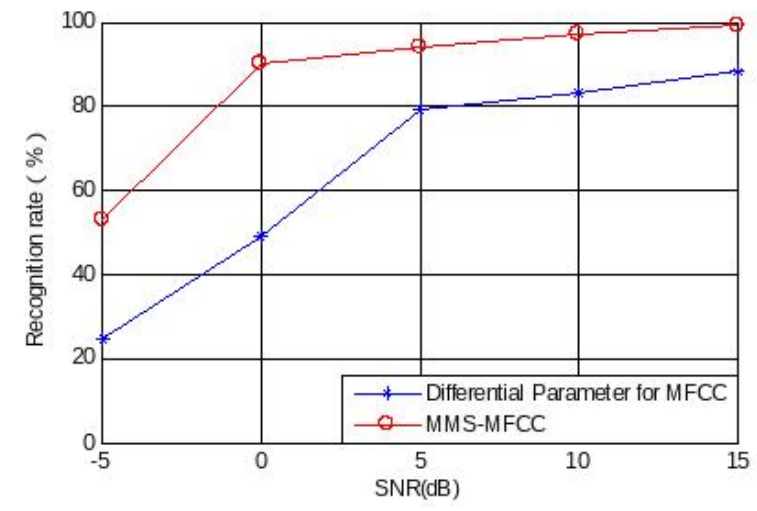

(b) The recognition curve under pink noise.

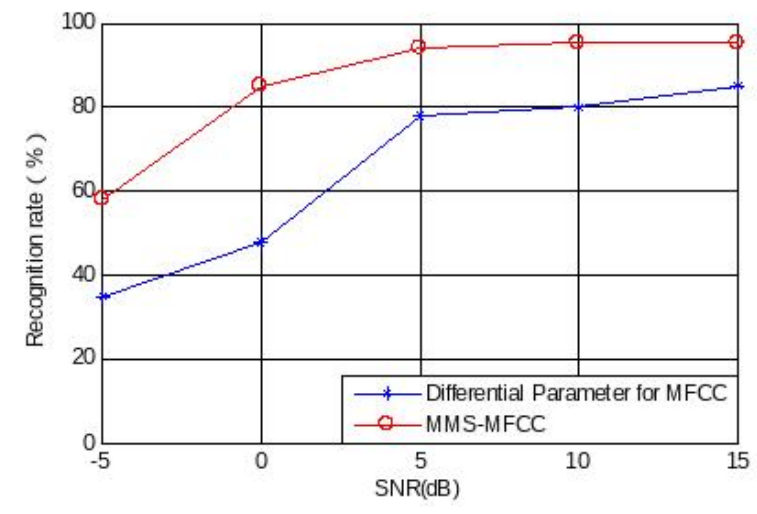

(c) The recognition curve under street noise.

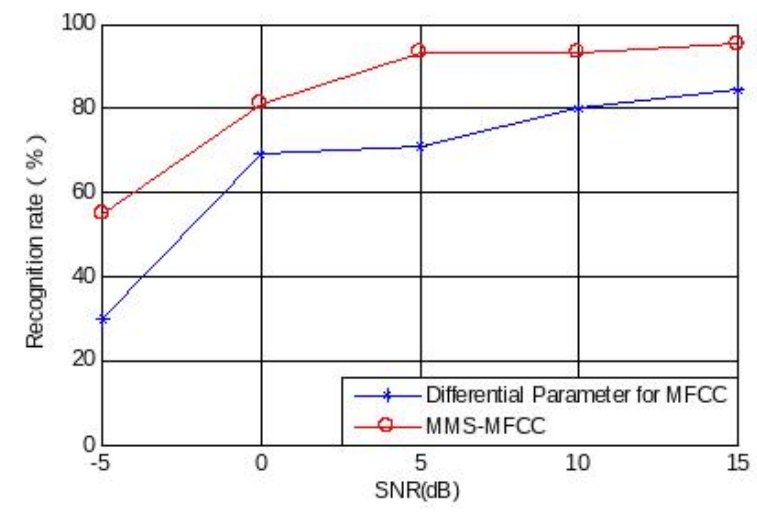

(d) The recognition curve under panzer noise.

Figure 5. The recognition performance curve under different SNR. 
61503038) and a grant from Education Department of Liaoning Province of China (No. L2013423).

\section{REFERENCES}

1 Jo, J., Yoo, H., and Park, I. C. Energy-Efficient floating-point MFCC extraction architecture for speech recognition systems, IEEE Trans. on Very Large Scale Integration Systems, 24(2), 754-758, (2016). http://dx.doi.org/tvlsi.2015.2413454

2 Jang, G. J., and Cho, H. Y. Efficient spectrum estimation of noise using line spectral pairs for robust speech recognition, Electronics Letters, 47(25), 1399-1401, (2011). http://dx.doi.org/10.1049/el.2011.2830

3 Taabish, G., Anand, S., and Sandeep, S. Comparative analysis of LPCC, MFCC and BFCC for the recognition of Hindi words using artificial neural networks, International Journal of Computer Applications, 101(12), 22-27, (2014). http://dx.doi.org/10.5120/17740-8271

4 Murty, K. S. R. and Yegnanarayana, B. Combining evidence from residual phase and MFCC features for speaker recognition, IEEE Trans. on Acoustics, Speech and Signal Processing, 13(1), 52-55, (2006). http://dx.doi.org/10.1109/lsp.2005.860538

5 Yang, L. D., Wang, J., Xie, X., and Kuang, J. M. Application of tucker decomposition in speech signal feature extraction, 2013 International Conference on Asian Language Processing, Urumqi, (2013). http://dx.doi.org/10.1109/ialp.2013.50

$6 \mathrm{Wu}$, Q., Zhang, L. Q., and Shi, G. C. Robust multifactor speech feature extraction based on gabor analysis, IEEE Trans. on Audio, speech, and Language Processing, 19(4), 927-936, (2011). http://dx.doi.org/10.1109/tasl.2010.2070495

7 Zou, X. Y., Zheng, W. Q., Wei, S., and Hong, L. Improved voice activity detection based on support vector machine with high separable speech feature vectors, The 19th international Conference on Digital Signal Processing, Hong Kong, (2014). http://dx.doi.org/10.1109/icdsp.2014.6900767

8 Deng, J. P., Bouchhard, M., and Yeap, T. H. Feature enhancement for noisy speech recognition with a time-variant linear predictive HMM structure, IEEE Trans. on Audio, speech, and Language Processing, 16(5), 891-899, (2008). http://dx.doi.org/10.1109/tasl.2004.924593

9 Ludovic, T., Philippe, G., and Brahim, C. D. Feature selection for robust automatic speech recognition: a temporal offset approach, International Journal of Speech Technology, 18(3), 395-404, (2015). http://dx.doi.org/10.1007/s10772-015-9276-6
10 Sharma, U., Maheshkar, S., and Mishra, A. N. Study of robust feature extraction techniques for speech recognition system, The International Conference on Futuristic Trends on Computational Analysis and Knowledge Management, Noida, (2015). http://dx.doi.org/10.1109/ablaze.2015.7154944

11 Rieken, D. W. and Fuhrmann, D. R. Generalizing MUSIC and MVDR for multiple noncoherent arrays, IEEE Trans. on Signal Processing, 52(9), 2396-2406, (2004). http://dx.doi.org/10.1109/tsp.2004.832153

12 Kaushik, M. Spectrum estimation, notch filters, and MUSIC, IEEE Trans. on Signal Processing, 53(10), 37273737, (2005). http://dx.doi.org/10.1109/tsp.2005.855395

13 Chen, X. D. and Agarwal, K. MUSIC algorithm for two-dimensional inverse problems with special characteristic of cylinders, IEEE Trans. on Antennas and Propagation, 56(6), 1808-1812, (2008). http://dx.doi.org/10.1109/tap.2008.923333

14 Smith, C. L., Browman, C. P., Mcgowan, R. S., and Kay, B. Extracting dynamic parameters from speech movement data, J. Acoust. Soc. Am., 93(3), 1580-1588, (1993). http://dx.doi.org/10.1121/1.406817

15 Yousefi, M. T. and Kschischang, F. R. Information transmission using the nonlinear fourier transform, part III: spectrum modulation, IEEE Trans. on Information Theory, 60(7), 4346-4369, (2014). http://dx.doi.org/10.1109/tit.2014.2321155

16 Sridhar, K. N., Kailash, P., and Mounya, E. A multistream feature framework based on bandpass modulation filtering for robust speech recognition, IEEE Trans. on Audio, Speech, and Language Processing, 21(2), 416-426, (2013). http://dx.doi.org/10.1109/tit.2014.2321155

17 Dharanipragada, S., Yapanel, U. H., and Rao, B. D. Robust feature extraction for continuous speech recognition using the MVDR spectrum estimation method, IEEE Trans. on Audio, speech, and Language Processing, 15(1), 224-234, (2007). http://dx.doi.org/10.1109/tasl.2006.876776

18 Yang, S. S., Ho, C. L. and Lee, C. M. HBP improvement in BP algorithm for an adaptive MLP decision feedback equalizer, IEEE Trans. on Circuits and Systems, 53(3), 240-244, (2006). http://dx.doi.org/10.1109/tcsii.2005.858494 